\title{
Comparison of Mechanical Axis and Dynamic Range Assessed with Weight Bearing Radiographs and Navigation System in Closed Wedge High Tibial Osteotomy
}

\author{
Dae Kyung Bae, MD, Jong Whan Lee, MD, Seong Jin Cho, MD, and Sang Jun Song, MD \\ Department of Orthopaedic Surgery, Kyung Hee University College of Medicine, Seoul, Korea
}

\begin{abstract}
Purpose: To compare navigation and weight bearing radiographic measurements of mechanical axis (MA) before and after closed wedge high tibial osteotomy (HTO) and to evaluate post-osteotomy changes in MA assessed during application of external varus or valgus force.

Materials and Methods: Data from 30 consecutive patients (30 knees) who underwent computer-assisted closed-wedge HTO were prospectively analyzed. Pre- and postoperative weight bearing radiographic evaluation of MA was performed. Under navigation guidance, pre- and post-osteotomy MA values were measured in an unloaded position. Any change in the post-osteotomy MA in response to external varus or valgus force, which was named as dynamic range, was evaluated with the navigation system. The navigation and weight bearing radiographic measurements were compared. Results: Although there was a positive correlation between navigation and radiographic measurements, the reliability of navigation measurements of coronal alignment was reduced after osteotomy and wedge closing. The mean post-osteotomy MA value measured with the navigation was $3.5^{\circ} \pm 0.8^{\circ}$ valgus in an unloaded position. It was $1.3^{\circ} \pm 0.8^{\circ}$ valgus under varus force and $5.8^{\circ} \pm 1.1^{\circ}$ valgus under valgus force. The average dynamic range was $> \pm 2^{\circ}$. Conclusions: Potential differences between the postoperative MAs assessed by weight bearing radiographs and the navigation system in unloaded position should be considered during computer-assisted closed wedge HTO. Care should be taken to keep the dynamic range within the permissible range of alignment goal in HTO.
\end{abstract}

Keywords: Knee, Osteotomy, Mechanical axis, Navigation, External force, Reliability

\section{Introduction}

High tibial osteotomy (HTO) can alter the mechanical axis (MA) of a limb so that the weight bearing load is transferred to healthy cartilage during the treatment of osteoarthritis with varus deformity $^{1-3)}$. In several studies, a proper postoperative MA was achieved using a cable, grid lines, a jig system, and a navigation

Received May 12, 2016; Revised July 24, 2016;

Accepted September 9, 2016

Correspondence to: Sang Jun Song, MD

Department of Orthopaedic Surgery, Kyung Hee University College of Medicine, 23 Kyunghee-daero, Dongdaemun-gu, Seoul 02447, Korea

Tel: +82-2-958-9489, Fax: +82-2-964-3865

E-mail: songsjun@khmc.or.kr

This is an Open Access article distributed under the terms of the Creative Commons Attribution Non-Commercial License (http://creativecommons.org/licenses/by-nc/4.0/) which permits unrestricted non-commercial use, distribution, and reproduction in any medium, provided the original work is properly cited. system $^{4-6)}$. Navigation systems provide reliable real-time intraoperative information and may increase the accuracy of $\mathrm{HTO}^{3,4,7)}$. The majority of previous studies reported a positive correlation between navigation and radiographic data ${ }^{4,8-11)}$. However, some studies provided equivocal data ${ }^{12,13)}$. Discrepancies of $>2^{\circ}$ between navigation and weight bearing radiographic measurements of a corrected alignment have been reported ${ }^{12,13)}$. This may be due to the difficulty of predicting a weight bearing alignment in a supine position under anesthesia, even with the use of a navigation system. Another possible reason might be postoperative changes in the tension of soft tissue surrounding the knee joint after $\mathrm{HTO}^{9,14)}$.

During computer-assisted closed-wedge HTO, we observed post-osteotomy changes in MA in response to external varus or valgus forces. This change in post-osteotomy MA in response to external forces was named "dynamic range". One question that arises is whether postoperative MA on weight bearing radio- 
graphs is still within the dynamic range of MA assessed by the navigation system.

The primary aim of this study was to analyze the reliability of navigation and weight bearing radiographic measurements of preoperative and postoperative MAs in patients with computerassisted closed wedge HTO. A secondary aim was to evaluate changes in MA in response to external varus or valgus forces by the navigation system and to compare with values measured on weight bearing radiographs. We hypothesized that there would be changes in reliability of the navigation and weight bearing radiographic measurements of the alignment and that post-osteotomy MA would exhibit a wide dynamic range under external forces.

\section{Materials and Methods}

\section{Patients}

Data were obtained from patients who underwent computerassisted closed wedge HTO between August 2012 and November 2013. The Vector Vision CT-free navigation system ver. 1.1 (BrainLAB, Heimstetten, Germany) was used for medial compartment osteoarthritis with a varus deformity $>10^{\circ}$. A miniplate staple (U\&I Co., Uijeongbu, Korea) was used as a fixative ${ }^{15}$. Informed consent was obtained from all patients, none of whom declined to participate. Thirty patients (30 knees; 17 left and 13 right) were included; their mean age was $57.7 \pm 5.5$ years (range, 46 to 68 years) and mean body mass index was $25.2 \pm 2.3 \mathrm{~kg} / \mathrm{m}^{2}$ (range, 20.5 to $30.3 \mathrm{~kg} / \mathrm{m}^{2}$ ). There were 26 females and 4 males.

\section{Radiographic Evaluation}

Radiographic measurements of the coronal alignment were performed using a full-length weight bearing anteroposterior radiograph of the leg, including the hip, knee, and ankle (i.e., an orthoroentgenogram). Lateral radiographs of the knee were obtained and reviewed to assess the tibial posterior slope angle (PSA). High-quality pre- and postoperative X-rays were obtained for all patients, and all measurements were performed using the standardized picture-acquiring and communication system $(\mathrm{PACS})^{16)}$. Coronal alignment (expressed as MA and MA\% [the proportion of the MA that bisects the total width of the tibial width]) (Fig. 1A) values were evaluated radiographically. The femoral MA was defined as the line adjoining the centers of the hip and distal femoral notch. The tibial MA was defined as the line adjoining the centers of the tibial intercondylar eminences and the talus. The MA was defined as the angle between the femoral and tibial MAs (Fig. 1B) ${ }^{4,6}$. Postoperative radiographic evaluation was performed using the identical method 3 months after surgery when the patient could stand with complete weight bearing. The PSA was measured with a reference line connecting the center of the medullary canal $10 \mathrm{~cm}$ and $20 \mathrm{~cm}$ distal to the tibial plateau. Preoperative PSA was defined as the angle between the reference line and a line connecting the anterior and posterior borders of the medial tibial plateau. To reduce observation bias,
A

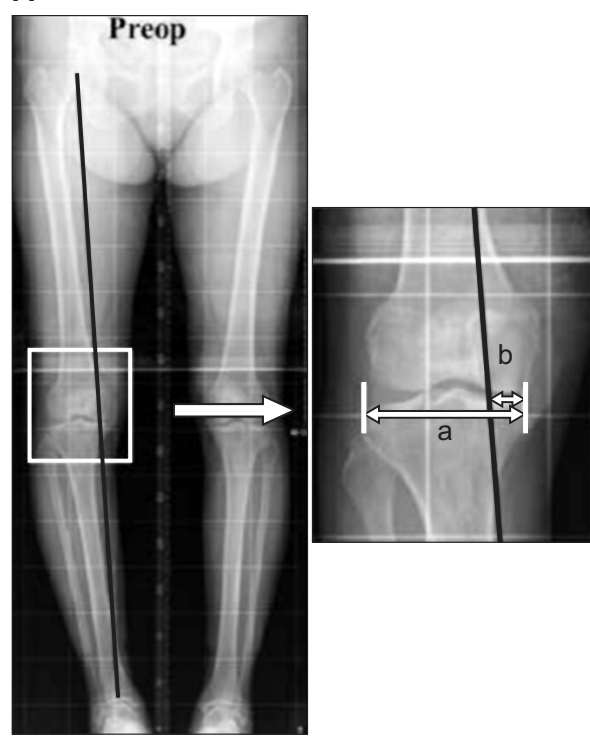

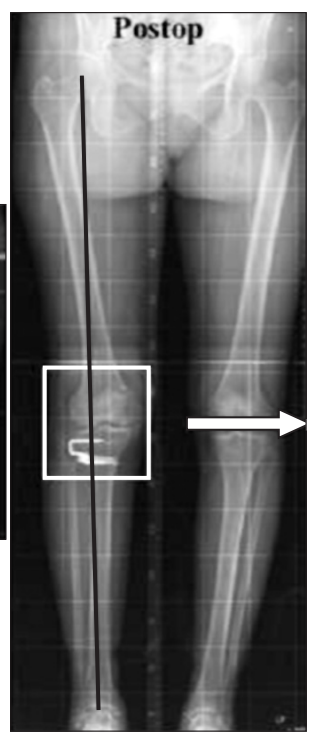

B

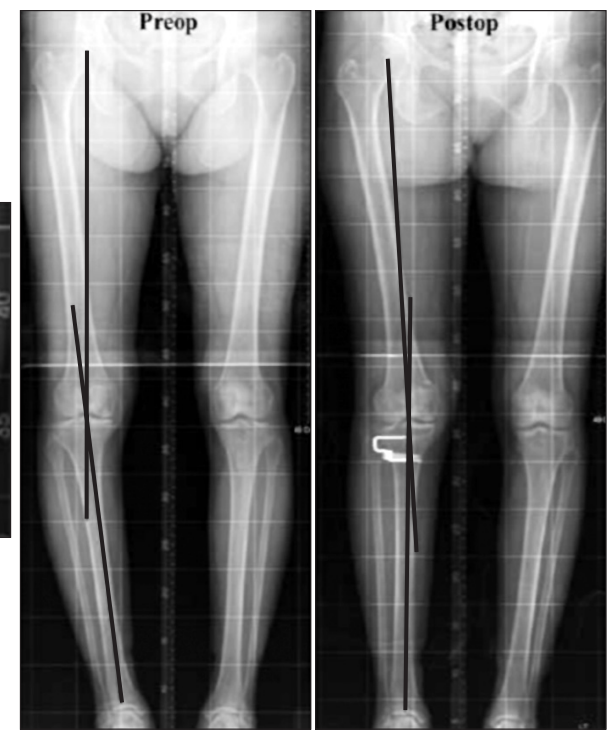

Fig. 1. Radiographic measurement of preoperative (preop) and postoperative (postop) mechanical axis (MA) and the percentage of mechanical axis (MA\%). (A) The MA\% was evaluated on the orthoroentgenogram and expressed in percentage [(b/a) $\times 100]$. (B) The MA was defined as the angle between femoral and tibial mechanical axes on the orthoroentgenogram. a: the width of tibia plateau, b: the distance from the medial border of the medial tibial condyle to the point at which the mechanical axis intersects the knee joint line. 
two independent investigators repeatedly performed all radiographic measurements. The intra- and inter-observer reliabilities of all measurements were assessed using intraclass correlation coefficients (ICC) ${ }^{17)}$, which were $>0.8$ for all measures.

\section{Measurement by the Navigation System}

Under navigation guidance, the MA was measured before osteotomy. Post-osteotomy MA and MA\% values were measured following wedge closing and fixation. After assessing post-osteotomy MA in the unloaded position, we evaluated changes in postosteotomy MA under conditions of external varus or valgus force (Fig. 2).

\section{Statistical Analysis}

Measurement data obtained under navigation guidance were compared with radiographic data using paired $t$-tests. The reliability of navigation and radiographic measurements was assessed using Pearson correlation analysis. ICC values $>0.8$ were considered very strong; values between 0.6 and 0.8 were moderately strong, values between 0.3 and 0.6 were fair, and values $<0.3$ were weak $^{18)}$. In addition to data obtained in the unloaded position, mean post-osteotomy MA and MA\% values, assessed using the navigation system during application of external varus or valgus force, were also compared to the radiographically measured values. They were directly compared in each knee to assess whether postoperative MA values on weight bearing radiographs are within the dynamic range measured using the navigation system during application of external forces. Patients were categorized according to the difference between their postoperative MA values on radiographs and post-osteotomy MA values in the unloaded position obtained using the navigation system. When the difference was between $-2^{\circ}$ and $2^{\circ}$, patients were assigned to group $\mathrm{N}$ (no difference). When the difference was $<-2^{\circ}$ or $>2^{\circ}$, patients were assigned to group $\mathrm{D}$ (reduced valgus alignment on weight bearing radiographs) or I (increased valgus alignment on weight bearing radiographs). Preoperative and postoperative MA and PSA values on radiographs, and pre- and post-osteotomy MA values in the navigation system, were compared between groups $\mathrm{N}$ and $\mathrm{D}$ (Student $t$-test) to assess whether correction angle or PSA influences changes in MA on weight bearing radiographs.

Statistical analysis was performed using the SPSS ver. 18.0 (SPSS Inc., Chicago, IL, USA). A value of $\mathrm{p}<0.05$ was taken to indicate statistical significance.

\section{Surgical Method}

A transverse skin incision extending from the tibial tubercle to the fibular neck is made, and the anterior tibialis muscle is subperiosteally dissected ${ }^{4)}$. Partial resection of the fibular head is made to avoid the tethering effect of the fibula during wedge closing in every knee. Two separate reference bases are fixed to the distal femur and to the middle tibia with a pair of half-pins. Eight kinematic and anatomic landmarks are registered. These include the kinematic hip center, medial and lateral malleoli, medial and lateral tibial plateau points, medial and lateral femoral epicondyles, and AP tibial rotation. The surgeon should take care to make the start and end points to be $15 \mathrm{~mm}$ distal to the tibial plateau. The far cortical hinge is tailored to be $3 \mathrm{~mm}$ in order to help the surgeon to close the osteotomy surface easily. The tibial posterior slope can be fine-tuned in the planned osteotomy according to the the PSA measured on the preoperative radiograph. The navigation system provides information about the deformity,

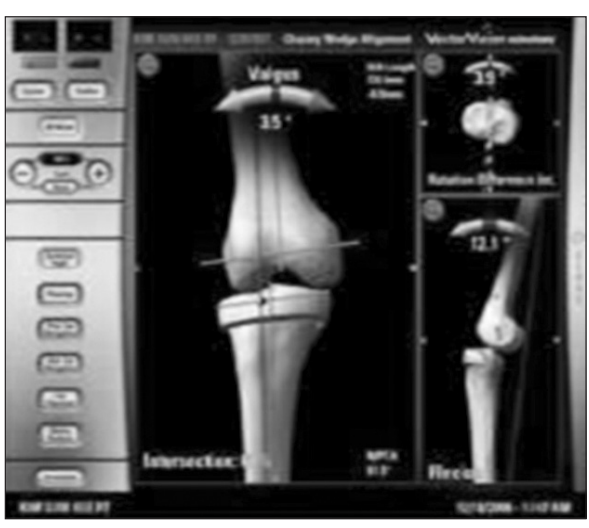

In unladed position

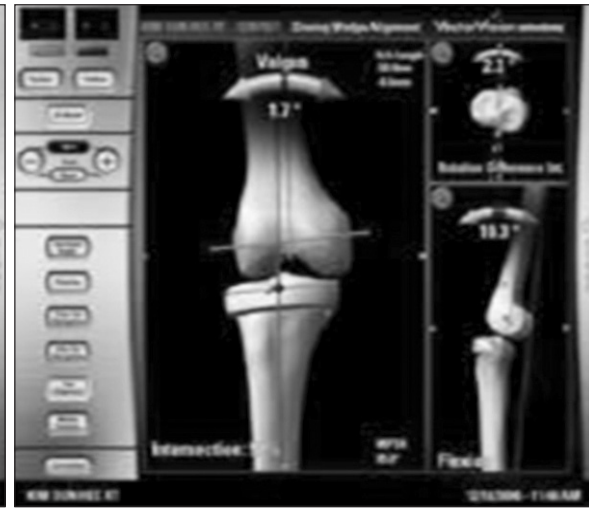

Under varus force

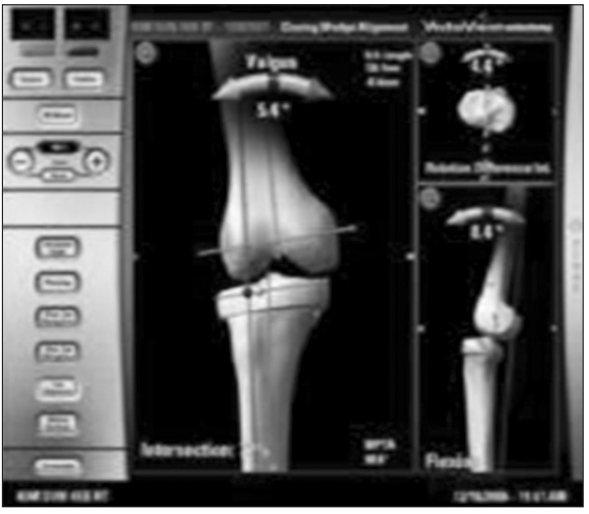

Under valgus force

Fig. 2. The alteration of postosteotomy mechanical axis (MA) and percentage of mechanical axis (MA\%) on navigation under external force. In this knee, the post-osteotomy MA and MA $\%$ were $3.5^{\circ}$ and $62 \%$, respectively, in the unloaded position. Those values were decreased to $1.7^{\circ}$ and $52 \%$, respectively, under varus force, and increased to $5.4^{\circ}$ and $72 \%$, respectively, under valgus force. 
level of osteotomy, correction angle, and wedge size. For the optimum degree of correction, the postoperative MA\%, which can be obtained via the navigation system, should be $62 \%^{6,199}$. A precalibrated navigated drill guide is used to place two K-wires in the proximal plane of the osteotomy. Another two K-wires are inserted in the distal plane of the osteotomy in the same manner. This 4 pin technique helps to make the anterior and posterior lengths of the wedge symmetric and to minimize the change in $\mathrm{PSA}^{4}$. The proximal and distal osteotomies are carried out using a sharp electric saw over two K-wires. The wedge is removed and the medial far cortex is then carefully decorticated using a sharp electric saw or an osteotome. A valgus force is applied slowly to the extremity until the proximal and distal osteotomy surfaces are firmly attached. Then, the osteotomy site is rigidly fixed using a miniplate staple of appropriate size ${ }^{15}$.

The final alignment or post-HTO MA and MA\% on the computer screen were confirmed in the unloaded position. The change of post-osteotomy MA and MA\% under external varus or valgus force was also evaluated using the navigation system (Fig. 2). A systematic rehabilitation protocol was used: straight leg rais-

Table 1. Correlation between Navigation System and Radiographic Measurements of Coronal Alignment

\begin{tabular}{ccccc}
\hline \multirow{2}{*}{ Variable } & Navigation & Radiograph & \multicolumn{2}{c}{ Correlation analysis } \\
\cline { 5 - 6 } & & & ICC & p-value \\
\hline Preoperative & & & & \\
MA $\left(^{\circ}\right)$ & Varus $9.8 \pm 2.5$ & Varus $8.5 \pm 2.7$ & 0.793 & $<0.001$ \\
MA\% (\%) & $10.7 \pm 11.7$ & $11.8 \pm 12.8$ & 0.986 & $<0.001$ \\
Postoperative & & & & \\
MA $\left(^{\circ}\right)$ & Valgus 3.5 \pm 0.8 & Valgus $2.7 \pm 1.5$ & 0.344 & 0.039 \\
MA\% (\%) & $62.2 \pm 0.9$ & $58.5 \pm 8.7$ & 0.312 & 0.048 \\
\hline
\end{tabular}

ICC: intraclass correlation coefficient, MA: the angle between the femoral and tibial mechanical axes, MA\%: the percentage at which the mechanical axis bisects the total width of the tibia. ing exercises were started 3 days postoperatively; partial weight bearing was started 1 week postoperatively; and full weight bearing without crutches was started at 6-12 weeks based on the patient's condition.

\section{Results}

After computer-assisted closed wedge HTO, varus deformity was corrected according to both navigation and radiographic data (Table 1). There was a positive correlation between navigation and radiographic measurements of pre- and postoperative MA and MA\%. According to ICC values, the preoperative correlation between measures was strong, and the postoperative correlation was fair (Table 1). The average post-osteotomy MA and MA\% assessed using the navigation system were $3.5^{\circ} \pm 0.8^{\circ}$ valgus and $62.2 \pm 0.9 \%$, respectively, in the unloaded position. The post-osteotomy MA and MA\% were changed by external varus and valgus forces. The average change of MA was more than $\pm 2^{\circ}$ (Table 2, Fig. 3). Although the mean post-osteotomy MA and MA\% obtained using the navigation system in the unloaded position were correlated to those obtained on radiography (Table 2), differences of $>2^{\circ}$ were observed in several knees (Fig. 3). When patients were categorized according to the difference of postoperative MA values measured on weight bearing radiographs and using the navigation system in the unloaded position, there were 22 knees in group $\mathrm{N}$ with the difference between $-2^{\circ}$ and $2^{\circ}, 7$ knees in group $\mathrm{D}$, and one knee in group I. The radiography-derived values were typically lower than navigation-derived values in the unloaded position in group D (Fig. 3). Preoperative MA and PSA values on the radiograph did not differ between groups $\mathrm{N}$ and D (Table 3). Pre- and post-osteotomy MA values by the navigation system were also similar between the two groups, including post-osteotomy MA values obtained under varus and valgus forces. The average postoperative MA on weight bearing

Table 2. Comparison of Post-osteotomy MA and MA\% in Unloaded Position and Under Varus or Valgus External Force on Navigation and in Weight Bearing Radiograph

\begin{tabular}{|c|c|c|c|c|}
\hline Postoperative & Unloaded position & Varus force & Valgus force & Radiograph \\
\hline $\operatorname{MA}\left({ }^{\circ}\right)$ & Valgus $3.5 \pm 0.8$ & Valgus $1.3 \pm 0.8$ & Valgus $5.8 \pm 1.1$ & Valgus $2.7 \pm 1.5$ \\
\hline ICC & 0.344 & 0.270 & 0.254 & \\
\hline MA\% & $62.2 \pm 0.9$ & $50.7 \pm 4.5$ & $74.8 \pm 0.9$ & $58.5 \pm 8.7$ \\
\hline ICC & 0.312 & 0.027 & 0.218 & \\
\hline
\end{tabular}

MA: the angle between the femoral and tibial mechanical axes, MA\%: the percentage at which the mechanical axis bisects the total width of the tibia, ICC: intraclass correlation coefficient. 


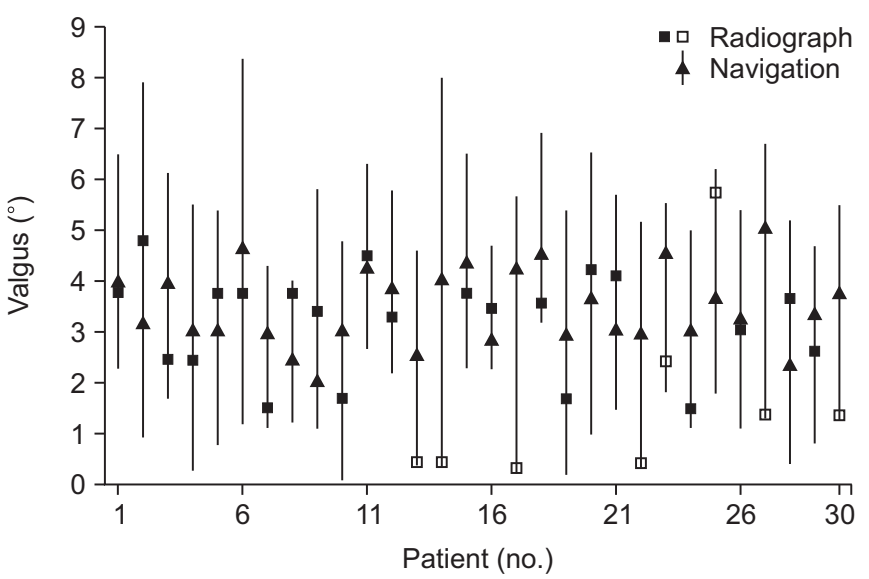

Fig. 3. Post-osteotomy mechanical axis (MA) values on navigation and weight bearing radiographs. Triangles indicate post-osteotomy MA values on navigation in the unloaded position; lines indicate dynamic range during application of varus and valgus force. Squares indicate postoperative MA values during weight-bearing on radiographs. In total, eight knees exhibited a difference of $>2^{\circ}$ between radiography- and navigation-derived values in the unloaded position (radiographic values are denoted by open squares).

radiographs was valgus $3.1^{\circ}$ in group $\mathrm{N}$ and valgus $0.9^{\circ}$ in group $\mathrm{D}$, respectively, although navigated values were similar in both groups for postoperative MA (Table 3 ).

\section{Discussion}

The most important finding of this study was that the reliability of MA and MA\% values measured using the navigation system decreased after osteotomy and wedge closing, although there was a positive correlation between navigation and radiographic measurements. Based on the assumption that accurate correction involves a weight bearing axis that intersects the tibial plateau at $50 \%-70 \%$ of the cross sectional tibial width ${ }^{6}$, navigation measurements obtained in a neutral position or during application of external force may be decreased compared to measurements obtained radiographically during standing. According to ICC values, the strong preoperative correlation between navigation and radiographic measurements decreased to "fair" postoperatively. The discrepancy of MA measurement between weight bearing and non-weight bearing condition may be a critical problem in HTO irrespective of the use of a navigation system. The weight bearing postoperative MA is difficult to predict accurately during osteotomy in the supine position, and this can result in the correction error of alignment ${ }^{20)}$. The present study showed that the reliability of non-weight bearing navigation measurements of MA decreased after osteotomy. Therefore, we think that careful assessment of MA by the navigation system will be necessary.
Table 3. Comparison of Navigation System and Radiographic Measurements of Coronal Alignment between Group N and Group D

\begin{tabular}{lccc}
\hline \multicolumn{1}{c}{ Measurement } & Group N $(\mathrm{n}=22)$ & Group D $(\mathrm{n}=7)$ & $\mathrm{p}$-value \\
\hline $\begin{array}{l}\text { Preoperative }\left(^{\circ}\right) \\
\text { Radiograph }\end{array}$ & & & \\
MA & $11.3 \pm 3.0$ & $11.7 \pm 2.8$ & 0.780 \\
PSA & & & \\
Navigation & Varus $9.8 \pm 2.7$ & Varus 9.9 \pm 1.8 & 0.888 \\
MA & & & \\
Postoperative $\left(^{\circ}\right)$ & & & \\
Navigation & & & \\
MA in unloaded position & Valgus 3.4 \pm 0.7 & Valgus 3.9 \pm 0.9 & 0.119 \\
MA under varus force & Valgus $1.3 \pm 0.9$ & Valgus $1.3 \pm 0.6$ & 0.855 \\
MA under valgus force & Valgus $5.7 \pm 1.1$ & Valgus 5.9 51.1 & 0.712 \\
Radiograph & & & \\
MA & Valgus 3.1 \pm 1.0 & Valgus $0.9 \pm 0.8$ & $<0.001$ \\
PSA & $8.6 \pm 3.5$ & $9.2 \pm 1.7$ & 0.515 \\
\hline
\end{tabular}

MA: the angle between the femoral and tibial mechanical axes, PSA: posterior tibial slope angle.

Fibular management and wedge closing during closed wedge HTO might affect alterations in soft tissue tension and postoperative alignment during standing assessed by radiographs. Lee et al. ${ }^{13)}$ also reported that the reliability of navigation measurements of alignment after surgery was decreased following computerassisted open wedge HTO, possibly because superficial medial collateral ligament (MCL) injury sustained during the procedure could cause valgus instability on weight bearing radiographs. Kendoff et al.9. reported that application of a simulated weight bearing force altered $\mathrm{MA}$ values by up to $2.5^{\circ}$ during computerassisted open wedge $\mathrm{HTO}$, due to changes in soft tissue tension, including functional lengthening of the lateral collateral ligament (LCL) exterior to the hinge axis, and MCL release. We hypothesized that alterations in soft tissue tension, and its effects on weight bearing alignment, would be detected during computerassisted closed wedge HTO. Fibular management, including proximal tibiofibular dissociation and partial fibular head resection, could cause LCL laxity after wedge closing, decreasing the valgus correction angle measured by the navigation system under varus external force.

A second important finding was that post-osteotomy MA and MA\% values measured by the navigation system changed under external varus and valgus forces and were associated with a wide dynamic range compared to the unloaded position (Fig. 3). The change in MA under external force may be explained by functional MCL laxity external to the hinge axis or LCL laxity after 
closed osteotomy. The average dynamic range of post-osteotomy MA was $> \pm 2^{\circ}$ on navigation, and varied between patients (Fig. 3). Postoperative MA values on weight bearing radiographs were within the dynamic range of postoperative MA values obtained under external force by the navigation system. Although weight bearing radiographic measurements were similar to navigation measurements in the unloaded position in the majority of patients, they were decreased in seven knees, and were similar to navigation measurements obtained during the application of external varus force (Table 3, Fig. 3). This finding accords with previous studies ${ }^{4,14,21)}$ describing a "spring-back" effect from conditions of LCL laxity and under-correction of postoperative MA after closed wedge HTO. The ideal postoperative MA angle is between $2^{\circ}-6^{02,22)}$, therefore, surgeons will be able to aim for a corrected angle within this range during the computer-assisted closed wedge HTO. Under navigation guidance, post-osteotomy MA values in both the unloaded position, and under external varus force, may be corrected to resolve the unpredictability associated with LCL laxity and under-correction.

Finally, we observed neither group difference (N vs. D) in any confounding variable nor any factor predicting reductions in MA correction. Kendoff et al. ${ }^{9)}$ demonstrated significant shifts in MA during external loading and weight bearing, and further reported that degree of correction, applied magnitude, and ligament integrity all affected the alteration of postoperative MA values under external loading conditions. In the present study there was no group difference ( $\mathrm{N}$ vs. $\mathrm{D}$ ) in the severity of preoperative varus deformity, correction angle, or alterations in PSA. However, postoperative MA and MA\% values on weight bearing radiographs differed between the two groups.

This study has several limitations. First, only closed wedge HTO was included due to our surgical preference. Recognition of changes in postoperative MA values, during weight bearing or under external force conditions during open wedge HTO, is also required to understand changes in correction angle during postoperative weight bearing and in the context of changes in soft-tissue tension. Second, the characteristics of our prospective cohort differed from those of Western HTO candidates, in whom the gender distribution of osteoarthritis and obesity prevalence may be important factors. Such differences must be considered before generalizing our data to other populations. Third, we did not perform any clinical evaluations. Assuming that proper postoperative alignment results in clinical satisfaction, we focused on the accuracy and reliability of navigated and radiographic measurements of MA. Fourth, the accuracy of percutaneous registration during computer-assisted HTO is limited. We marked landmarks before registration, after careful consideration of surface anatomy, to ensure accurate registration. Fifth, preoperative MA was not assessed under external varus and valgus force by the navigation system. It was difficult to explain the reason for reduced reliability of the measurement of MA by the navigation system. However, the preoperative MA under external force was negligible in full extended position of the knees in most patients. Sixth, the methodological categorization was based on cut-off value of $\pm 2^{\circ}$ according to the difference of postoperative MA values between weight bearing radiograph and navigation in unloaded position. It was thought to have clinical relevance and be within the radiographic measurement accuracy to be detected. Finally, the accuracy of radiographic measurements was limited. Slight changes in projection angle and rotation or flexion of the knee may have affected radiographic measurements. Although computed tomography can accurately measure the alignment, its application is limited due to radiation exposure and inability to obtain weight bearing images. We aimed to acquire consistently high quality images in the neutral position and confirmed the intra- and inter-observer reliability of all measurements.

\section{Conclusions}

The reliability of navigation measurements of coronal alignment was reduced after osteotomy and wedge closing. Post-osteotomy MA values assessed by the navigation system were altered under the application of external forces and exhibited a dynamic range. Potential differences of postoperative MA between weight bearing radiograph and navigation in unloaded position should be considered during computer-assisted closed wedge HTO, and the dynamic range on navigation should be kept within the permissible range of alignment goal in HTO.

\section{Conflict of Interest}

No potential conflict of interest relevant to this article was reported.

\section{References}

1. Coventry MB, Ilstrup DM, Wallrichs SL. Proximal tibial osteotomy: a critical long-term study of eighty-seven cases. J Bone Joint Surg Am. 1993;75:196-201.

2. Lee DC, Byun SJ. High tibial osteotomy. Knee Surg Relat Res. 2012;24:61-9.

3. Maurer F, Wassmer G. High tibial osteotomy: does naviga- 
tion improve results? Orthopedics. 2006;29(10 Suppl):S130-2.

4. Bae DK, Song SJ, Yoon KH. Closed-wedge high tibial osteotomy using computer-assisted surgery compared to the conventional technique. J Bone Joint Surg Br. 2009;91:116471.

5. Kim SJ, Koh YG, Chun YM, Kim YC, Park YS, Sung CH. Medial opening wedge high-tibial osteotomy using a kinematic navigation system versus a conventional method: a 1-year retrospective, comparative study. Knee Surg Sports Traumatol Arthrosc. 2009;17:128-34.

6. Reising K, Strohm PC, Hauschild O, Schmal H, Khattab M, Sudkamp NP, Niemeyer P. Computer-assisted navigation for the intraoperative assessment of lower limb alignment in high tibial osteotomy can avoid outliers compared with the conventional technique. Knee Surg Sports Traumatol Arthrosc. 2013;21:181-8.

7. Hofmann AA, Wyatt RW, Beck SW. High tibial osteotomy: use of an osteotomy jig, rigid fixation, and early motion versus conventional surgical technique and cast immobilization. Clin Orthop Relat Res. 1991;(271):212-7.

8. Heijens E, Kornherr P, Meister C. The role of navigation in high tibial osteotomy: a study of 50 patients. Orthopedics. 2009;32(10 Suppl):40-3.

9. Kendoff D, Board TN, Citak M, Gardner MJ, Hankemeier S, Ostermeier S, Krettek C, Hüfner T. Navigated lower limb axis measurements: influence of mechanical weight-bearing simulation. J Orthop Res. 2008;26:553-61.

10. Lutzner J, Gross AF, Günther KP, Kirschner S. Reliability of limb alignment measurement for high tibial osteotomy with a navigation system. Eur J Med Res. 2009;14:447-50.

11. Yamamoto $Y$, Ishibashi $Y$, Tsuda E, Tsukada H, Kimura $Y$, Toh S. Validation of computer-assisted open-wedge high tibial osteotomy using three-dimensional navigation. Orthopedics. 2008;31(10 Suppl 1).

12. Kyung BS, Kim JG, Jang KM, Chang M, Moon YW, Ahn JH, Wang JH. Are navigation systems accurate enough to predict the correction angle during high tibial osteotomy? Comparison of navigation systems with 3-dimensional computed tomography and standing radiographs. Am J Sports Med. 2013;41:2368-74.

13. Lee DH, Nha KW, Park SJ, Han SB. Preoperative and postoperative comparisons of navigation and radiologic limb alignment measurements after high tibial osteotomy. Arthroscopy. 2012;28:1842-50.

14. Shaw JA, Dungy DS, Arsht SS. Recurrent varus angulation after high tibial osteotomy: an anatomic analysis. Clin Orthop Relat Res. 2004;(420):205-12.

15. Bae DK, Mun MS, Kwon OS. A newly designed miniplate staple for high tibial osteotomy. Bull Hosp Jt Dis. 1997;56: 167-70.

16. Fowler JR, Ilyas AM. The accuracy of digital radiography in orthopaedic applications. Clin Orthop Relat Res. 2011;469: 1781-4.

17. Lachin JM. The role of measurement reliability in clinical trials. Clin Trials. 2004;1:553-66.

18. Chan YH. Biostatistics 104: correlational analysis. Singapore Med J. 2003;44:614-9.

19. Fujisawa Y, Masuhara K, Shiomi S. The effect of high tibial osteotomy on osteoarthritis of the knee: an arthroscopic study of 54 knee joints. Orthop Clin North Am. 1979;10:585608.

20. Ogata K, Yoshii I, Kawamura H, Miura H, Arizono T, Sugioka Y. Standing radiographs cannot determine the correction in high tibial osteotomy. J Bone Joint Surg Br. 1991;73:92731.

21. Gaasbeek RD, Nicolaas L, Rijnberg WJ, van Loon CJ, van Kampen A. Correction accuracy and collateral laxity in open versus closed wedge high tibial osteotomy: a one-year randomised controlled study. Int Orthop. 2010;34:201-7.

22. Jakob RP, Jacobi M. Closing wedge osteotomy of the tibial head in treatment of single compartment arthrosis. Orthopade. 2004;33:143-52. 